\title{
Post-Trauma Immunological Disruptions: A Review
}

\section{Purva Mathur*}

Department of Laboratory Medicine, Jai Prakash Narain Apex Trauma Centre, All India Institute of Medical Sciences, New Delhi, India

*Corresponding author: Purva Mathur, Department of Laboratory Medicine, Jai Prakash Narain Apex Trauma Centre, All India Institute of Medical Sciences, New Delhi, 110029, India, Tel: 26189000; 26188000; Fax: 91-11-26106826; E-mail: purvamathur@yahoo.co.in

Rec date: Feb 03, 2014, Acc date: Apr 13, 2014, Pub date: Apr 15, 2014

Copyright: (C) 2014 Mathur P. This is an open-access article distributed under the terms of the Creative Commons Attribution License, which permits unrestricted use, distribution, and reproduction in any medium, provided the original author and source are credited.

\begin{abstract}
Accidents and trauma are one of the world's most serious but neglected health problems. The fast moving transportation systems, unprecedented and unplanned urbanization and changing social patterns have contributed to the global increase in the incidence of trauma. Traffic accidents are an endemic disease which affects mainly the young adults in the economically productive age groups and are the leading cause of death in persons under 44 years of age. Globally, $26 \%$ of all deaths in the age group of $15-44$ years are due to injuries. The national vital registration system registers around 18 million deaths annually due to injuries worldwide. In Developing countries, trauma is a major problem, due to a very high incidence of vehicular accidents, other accidental injuries, crime and violence. Rising population, urbanization, industrialization and a drastic rise in vehicular transport has contributed to an annual increase in road traffic accidents by $3 \%$ in India alone. An accident death is reported every 1.9 minutes in India; in 1997, $10.1 \%$ of all deaths in India were due to accidents and injuries. Despite of this, trauma care is still at a developmental stage in our country. Thus, there is a growing population of traumatized patients requiring highly sophisticated and specialized care. Because most of these patients are young adults, with no underlying illness, there is a great concern to save these patients.
\end{abstract}

Keywords: Multidisciplinary trauma centers; Post-trauma complications; Immunological disturbances

\section{Introduction}

Accidents and trauma are one of the world's most serious but neglected health problems. The fast moving transportation systems, unprecedented and unplanned urbanization and changing social patterns have contributed to the global increase in the incidence of trauma. Traffic accidents are an endemic disease which affects mainly the young adults in the economically productive age groups and are the leading cause of death in persons under 44 years of age. Globally, $26 \%$ of all deaths in the age group of 15-44 years are due to injuries. The national vital registration system registers around 18 million deaths annually due to injuries worldwide.

In Developing countries, trauma is a major problem, due to a very high incidence of vehicular accidents, other accidental injuries, crime and violence. Rising population, urbanization, industrialization and a drastic rise in vehicular transport has contributed to an annual increase in road traffic accidents by $3 \%$ in India alone. An accident death is reported every 1.9 minutes in India; in 1997, $10.1 \%$ of all deaths in India were due to accidents and injuries. Despite of this, trauma care is still at a developmental stage in our country [1-6].

Thus, there is a growing population of traumatized patients requiring highly sophisticated and specialized care. Because most of these patients are young adults, with no underlying illness, there is a great concern to save these patients.

\section{Post-Trauma Complications and Mortality}

With the establishment of multidisciplinary Trauma Centers, the initiation of Advanced Trauma Life Support (ATLS) programs and advancements in intensive care, the management of major contributors of trauma associated mortality like hemorrhage, circulatory collapse and respiratory failure has greatly improved. However, patients admitted with trauma and injuries are highly prone to develop complications like sepsis, systemic inflammatory response syndrome (SIRS) and multiple organ dysfunction syndrome (MODS), which contributes to almost $80 \%$ of late deaths in them $[7,8]$.

Thus, mortality in trauma patients who survive the initial resuscitation is usually due to MODS or sepsis. Recent studies support that these complications are the result of activation or suppression of innate and adaptive immune responses. Although developments of modern trauma systems have significantly reduced early deaths, late organ complications in the surviving patients still present therapeutic challenges to surgeons and critical care physicians $[7,8]$.

\section{Post-Trauma Immunological Disturbances}

Immunological disturbances are the main drivers for post-trauma complications, morbidity and mortality. It is generally agreed that the disruptions of natural and adaptive immunity after traumatic and burn injuries are related to the increased susceptibility to SIRS, sepsis, and multiple organ failure (MOF) exhibited by those patients who survive beyond the initial resuscitation period. Furthermore, evidence suggests that trauma produces a bias towards a $\mathrm{TH} 2$ response and an exaggerated TH1 suppression, the degree of which may further predispose patients to sepsis, SIRS, and MOF. The body's immunologic response to trauma and burn injuries has been a subject of interest in recent years [8-29].

The post-trauma acute -phase immune response is mainly characterized by production and release of various pro-inflammatory cytokines like TNF- $\alpha$ and IL- $1 \beta$. Excessive production of these cytokines may predispose to SIRS. In order to balance the excessive cytokines, the body produces a compensatory response by releasing 
Page 2 of 4

anti-inflammatory cytokines (IL-10 and TGF- $\beta$ ), which is called compensatory anti-inflammatory response syndrome (CARS). Overproduction of either pro- or anti-inflammatory cytokines may result in organ dysfunction. These balances are depicted in figure 1. The inflammatory mediator increase could be used as early prognostic or diagnostic markers for complications. Serious injury also results in progressive suppression of adaptive immune response during the first week after trauma, which may contribute to the development of sepsis and MODS. When SIRS leads to MODS, mortality can increase up to $50-80 \%$. The overall mortality associated with sepsis ranges from $30-50 \%$ [8-29].

Studies to identify markers which could identify patients at risk for complications have identified decreased production of IL-12, IL-2 and IFN- $\gamma$, increased production of IL-10, IL-4 and IL-18, elevated sIL-2RA and the IL-6:IL- 10 ratio as the most promising prognostic markers. Currently, IL- 6 is regarded as being the most accurate marker for prognosis of SIRS, sepsis or MODS in trauma patients. However, different studies have observed markedly different levels of IL-6 posttrauma, depending on the time frames of sampling. The half-life of TNF- $\alpha$ is 20 minutes and that of IL-1 is 6 minutes, which may be a reason for the variable post-trauma levels found in different studies [8-29].

A drawback of most of the studies on post-trauma immunological disturbances has been that they have been done using serum/ plasma cytokine estimation, which may not depict the actual role of lymphocytic or monocytic dysfunction (Figure 1).

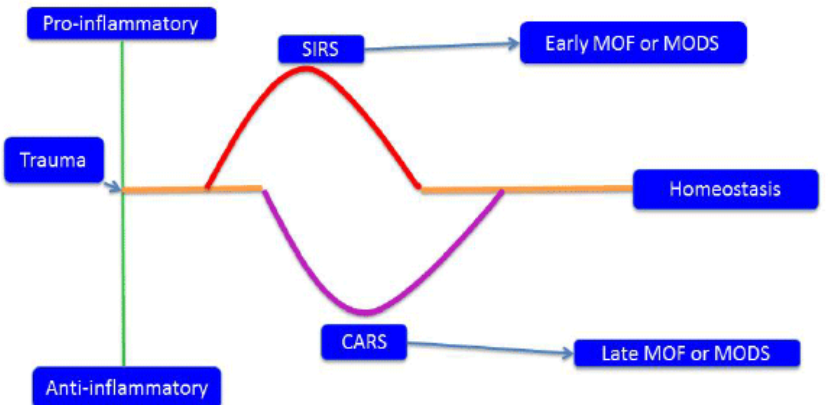

Figure 1: Post-injury inflammatory response, which may result in MODS

SIRS: Systemic Inflammatory Response Syndrome; CARS: Compensatory Anti-Inflammatory Response Syndrome; MOF: MultiOrgan Failure; MODS: Multiple Organ Dysfunction Syndromes

\section{TH Response Post-Trauma}

The method of intracellular staining of cytokine generation in activated T cells has been a powerful tool to differentiate TH1 and TH2 cells using flowcytometry. Studies have reported a clear increase in $\mathrm{TH} 2$ cells without a decrease in TH1 cells in severely burned patients after major surgery. In contrast, no increase in TH2 cells but a significant decrease in $\mathrm{TH} 1$ cells has been observed after minor surgeries $[10,12,29]$.

Some studies have postulated that in major trauma, a shift within the T-helper cell subsets and their specific cytokine products contributes to immune response dysregulation. However, because of extremely variable results in studies on cellular levels in humans, which strongly depend on cell type investigated and the experimental setting, those results do not allow us to currently include this postulate into current immune regulatory concepts. Frequently, these reported results were achieved under maximal non-physiologic stimulation in vitro; thus, the biological relevance has still to be proven $[10,12,29]$.

To achieve further knowledge about the role of TH1/TH2-type cytokines in the immune response after trauma, a recent study questioned the hypothesis that a shift toward TH2-type cytokine response occurs in the plasma of patients after major trauma that correlates with adverse outcome 29 . The authors investigated in a retrospective study 195 severely injured patients, both males and females during their ICU stay, post-trauma. TH1-type cytokines- IL-2, interferon $\gamma$, IL-12 and IL-18 and TH2-type cytokines IL-4, IL-10, and IL-11 were determined using ELISA in patients and in healthy controls. However, in their study, no TH1/TH2 shift was observed after major trauma. A drawback of this study was again that the authors used plasma levels of cytokines to monitor TH1/TH2 response $[10,12,29]$.

Spolarics et al. [12] studied the TH1/TH2 status in severely injured male patients by assessing the number of IL-4, IFN- $\gamma$ and TNF- $\alpha$ producing $\mathrm{CD} 4+$ and $\mathrm{CD} 8+\mathrm{T}$ cells. They tested the hypothesis that major trauma results in coordinated alterations in the cytokine producing activity of both monocytes and $\mathrm{T}$ cells and that initial monocyte and lymphocyte responses correlated with the severity of the inflammatory response as well as adverse clinical outcome. They observed that major trauma results in an early and marked decrease in monocyte cytokine producing activity, which may promote $\mathrm{T}$-cell commitment towards a TH-2 pattern early after trauma. Further, in their study, they found that the degree of alterations in monocytes and $\mathrm{T}$ cell responses on day 2 post injury correlates with the development of adverse clinical outcome and the duration of the inflammatory response. However, this study included only 12 patients [10,12,29].

\section{Role of Monocytes in Post-Trauma Immunological Response}

In order to salvage the trauma victims, attention has now shifted to the studying of functional aspects of monocytes during post-trauma phase. Monocyte activity may be the major driving force for posttrauma immunological disturbance. Cytokines are produced by macrophages and monocytes at the local site of injury and in the end organs $[11,12,15]$.

Spolarics et al. [12] showed that after LPS stimulation, the number of IL-12, TNF $\alpha$, IL-6, and IL-1 $\alpha$-producing monocytes were $40-70 \%$ lower in trauma patients. The number of IL-12-producing monocytes was markedly decreased in trauma patients who developed sepsis, and infections.

A recent study has shown that an immediate hyper activation of circulating monocytes is rapidly followed by a substantial paralysis of cell function, which correlated with the development of MODS [15].

Investigators have assayed monocyte activity in biological fluids from mixed or purified monocyte cultures or in whole blood challenged with LPS. Post-traumatic serum cytokine levels may represent only the peak of the cytokine release that takes place locally from diverse cell sources. Thus, the examination of monocytes function using LPS-stimulated whole blood via flowcytometric 
analysis may represent a more accurate assessment of changes in acute cellular function after severe trauma.

\section{Role of Monocyte Human Leucocyte Antigen-DR}

Since specific clinical signs of immune dysfunction are often absent in intensive care patients, studies have moved towards evaluation of biomarkers of immune suppression [30,31].

Diminished expression of Human Leucocyte Antigen DR on circulation monocytes (mHLA-DR) is widely accepted as a reliable indicator of immunosuppression in ICU patients. Very few studies have been done to assess the role of mHLA-DR in trauma patients. A literature review shows that except two studies, all the remaining preliminary studies were performed more than 10 years ago (i.e before the advent of advanced trauma life support [ATLS] protocols for the management of multiple-injured patients). These preliminary findings on mHLA-DR were based on limited numbers of patients and used non-standardized flowcytometry protocols [30,31].

The first study on trauma patients based on standardized protocols was published by Cheronet al in 2010 [31]. In this prospective, observational, 23-month study, the authors demonstrated that a persistently decreased expression of mHLA-DR post- trauma was associated with development of sepsis. Multivariate logistic regression analysis revealed that a slope of mHLA-DR expression between days 1-2 and days 3-4 below 1.2 was associated with the development of sepsis $[30,31]$.

In a very recent study (published in 2012), conducted over a period of 24 months in a trauma ICU, 100 consecutive severely injured patients were followed up to determine whether early measurement of IL-6 and IL-10 plasma concentrations (as markers of initial severity) along with mHL-DR recovery could improve the prediction of sepsis. In this study, IL- 6 and IL-10 were measured by ELISA. Of the 100 patients, 37 developed sepsis. The IL- 6 concentrations and slope of mHLA-DR expression between days 1-2 and 3-4 were significantly different between septic and non-septic patients [31].

It was concluded that a high IL-6 concentration and a persistent mHLA-DR decreased expression were found to be the best predictive marker for development of sepsis.

A limitation of this study was that only two cytokines were assessed and they were assessed only till day 4 post-trauma, with no attempt to analyze the intracellular cytokine productions.

\section{Role of Programmed Death -1 (PD-1) Related Molecules}

Programmed death -1 (PD-1) related molecules constitute a complex system of negative regulators involved in controlling T-cell response. This system is composed of PD-1 (CD-279) and its two ligands, PD-L1 (B7-H1, CD-274) and PD-L2 (B7-DC, CD-273). These molecules belong to the B7: CD-28 family. They are best understood relative to their role in viral infections and oncology 32 .

It has been proposed that pathogens and tumor cells may take advantage of this pathway to escape the host's immune defenses considering their immune regulatory properties. It is postulated that the PD-1 system could participate in sepsis-induced immune dysfunctions. It was recently shown that PD-1 knockout mice exhibited not only a greater capacity to clear bacteria, but more importantly, a lower mortality in response to experimental sepsis [32].
No study has been done on PD-1 related molecules in trauma patients.

\section{Role of IL-18}

IL-18, originally termed IFN- $\gamma$ inducing factor (IGIF), is a relatively new cytokine which shares structured homology with IL-1 family and some functional properties with IL-12 [33,34].

A few recent studies have found that IL-18 levels in plasma represent early and reliable markers for post-traumatic complications such as MODS and sepsis. However, these studies have also been done to assess extracellular cytokines in plasma $[33,34]$.

To conclude, it is now established that the immediate post-trauma mortality is due to hemorrhage, circulatory and respiratory collapse or severe brain injury. However, for those patients who survive the initial 4-5 days, immunological disruptions play a significant role in their progression to MODS, SIRS and Sepsis

\section{References}

1. World Health Organization Statistical Annex. World Report on Road Traffic Injury Prevention.

2. Mathur P (2008) Infections in traumatised patients: a growing medicosurgical concern. Indian J Med Microbiol 26: 212-216.

3. Mohta M, Kumar P, Mohta A, Bhardwaj R, Tyagi A, et al. (2006) Experiences with chest trauma. Where do we stand today. Ind J Crit Care Med 10: 25-28.

4. Joshipura MK, Shah HS, Patel PR, Divatia PA, Desai PM (2003) Trauma care systems in India. Injury 34: 686-692.

5. Sethi AK, Tyagi A (2001) Trauma untamed as yet. Trauma Care 11: 89-90.

6. National Crime Records Bureau, New Delhi (1999) Accidental deaths and suicide in India.

7. Caplan ES, Hoyt NJ (1985) Identification and treatment of infections in multiply traumatized patients. Am J Med 79: 68-76.

8. Lenz A, Franklin GA, Cheadle WG (2007) Systemic inflammation after trauma. Injury 38: 1336-1345.

9. Keel M, Trentz O (2005) Pathophysiology of polytrauma. Injury 36: 691-709.

10. Miller AC, Rashid RM, Elamin EM (2007) The "T" in trauma: the helper T-cell response and the role of immunomodulation in trauma and burn patients. J Trauma 63: 1407-1417.

11. Wutzler S, Maier M, Lehnert M, Henrich D, Walcher F, et al. (2009) Suppression and recovery of lps-stimulated monocyte activity after trauma is correlated with increasing injury severity: a prospective clinical study. J Trauma 66:1273-1280.

12. Spolarics Z, Siddiqi M, Siegel JH, Garcia ZC, Stein DS, et al. (2003) Depressed interleukin-12-producing activity by monocytes correlates with adverse clinical course and a shift toward Th2-type lymphocyte pattern in severely injured male trauma patients. Crit Care Med 31: 1722-1729.

13. Pape HC, Griensven MV, Hildebrand FF, Tzioupis CT, Sommer KL, et al. (2008) Systemic inflammatory response after extremity or truncal fracture operations. J Trauma 65: 1379-1384.

14. DeLong WG Jr, Born CT (2004) Cytokines in patients with polytrauma. Clin Orthop Relat Res : 57-65.

15. Kirchhoff C, Biberthaler P, Mutschler WE, Faist E, Jochum M, et al. (2009) Early down-regulation of the pro-inflammatory potential of monocytes is correlated to organ dysfunction in patients after severe multiple injury: a cohort study. Crit Care 13: R88.

16. Volk HD, Reinke P, Döcke WD (2000) Clinical aspects: from systemic inflammation to 'immunoparalysis'. Chem Immunol 74: 162-177. 
Page 4 of 4

17. Adrie C, Bachelet M, Vayssier-Taussat M, Russo-Marie F, Bouchaert I, et al. (2001) Mitochondrial membrane potential and apoptosis peripheral blood monocytes in severe human sepsis. Am J Respir Crit Care Med 164: 389-395.

18. Prussin C, Metcalfe DD (1995) Detection of intracytoplasmic cytokine using flow cytometry and directly conjugated anti-cytokine antibodies. J Immunol Methods 188: 117-128.

19. Gebhard F, Pfetsch H, Steinbach G, Strecker W, Kinzl L, et al. (2000) Is interleukin 6 an early marker of injury severity following major trauma in humans? Arch Surg 135: 291-295.

20. Rose S, Marzi I (1998) Mediators in polytrauma--pathophysiological significance and clinical relevance. Langenbecks Arch Surg 383: 199-208.

21. Dimopoulou I, Orfanos S, Kotanidou A, Livaditi O, GiamarellosBourboulis E, et al. (2008) Plasma pro- and anti-inflammatory cytokine levels and outcome prediction in unselected critically ill patients. Cytokine 41: 263-267.

22. Maier B, Lefering R, Lehnert M, Laurer HL, Steudel WI, et al. (2007) Early versus late onset of multiple organ failure is associated with differing patterns of plasma cytokine biomarker expression and outcome after severe trauma. Shock 28: 668-674.

23. Pape HC, Remmers D, Grotz M, Kotzerke J, von Glinski S, et al. (1999) Reticuloendothelial system activity and organ failure in patients with multiple injuries. Arch Surg 134: 421-427.

24. Donnelly TJ, Meade P, Jagels M, Cryer HG, Law MM, et al. (1994) Cytokine, complement, and endotoxin profiles associated with the development of the adult respiratory distress syndrome after severe injury. Crit Care Med 22: 768-776.

25. Jung T, Schauer U, Heusser C, Neumann C, Rieger C (1993) Detection of intracellular cytokines by flow cytometry. J Immunol Methods 159: 197-207.

26. He Z, Wang S, Su Z, Huang Y, Yang J (1999) [Changes of interleukin-2 and soluble interleukin-2 receptor serum level and multiple organ dysfunction syndrome in severely traumatic patients]. Zhonghua Wai Ke Za Zhi 37: 492-493.

27. Lyons A, Goebel A, Mannick JA, Lederer JA (1999) Protective effects of early interleukin 10 antagonism on injury-induced immune dysfunction. Arch Surg 134: 1317-1323.

28. Pape HC, Tsukamoto T, Kobbe P, Tarkin I, Katsoulis S, et al. (2007) Assessment of the clinical course with inflammatory parameters. Injury 38: 1358-1364.

29. Heizmann O, Koeller M, Muhr G, Oertli D, Schinkel C (2008) Th1- and Th2-type cytokines in plasma after major trauma. J Trauma 65: 1374-1378.

30. Gouel-Chéron A, Allaouchiche B, Guignant C, Davin F, Floccard B, et al. (2012) Early interleukin-6 and slope of monocyte human leukocyte antigen-DR: a powerful association to predict the development of sepsis after major trauma. PLoS One 7: e33095.

31. Cheron A, Floccard B, Allaouchiche B, Guignant C, Poitevin F, et al. (2010) Lack of recovery in monocyte human leukocyte antigen-DR expression is independently associated with the development of sepsis after major trauma. Crit Care 14: R208.

32. Guignant C, Lepape A, Huang X, Kherouf H, Denis L, et al. (2011) Programmed death-1 levels correlate with increased mortality, nosocomial infection and immune dysfunctions in septic shock patients. Crit Care 15: R99.

33. Mommsen P, Frink $\mathrm{M}$, Pape $\mathrm{C} \mathrm{H}$, Griensven van $\mathrm{M}$, Probst $\mathrm{C}$, et al (2009) Elevated systemic IL-18 and neopterin levels are associated with posttraumatic complication among patients with multiple injuries: A prospective cohort study. InjuryInt. J. Care Injured 40 528-534.

34. Oberholzer A, Steckholzer U, Kurimoto M, Trentz O, Ertel W (2001) Interleukin-18 plasma levels are increased in patients with sepsis compared to severely injured patients. Shock 16: 411-414. 